\title{
Article \\ Self-Similarity Properties of Complex Quasi-Periodic Fibonacci and Cantor Photonic Crystals
}

\author{
Aleksander Augustyniak $^{1}\left(\mathbb{D}\right.$, Mariusz Zdanowicz ${ }^{2} \mathbb{D}$ and Tomasz Osuch ${ }^{1,2, *(D)}$ \\ 1 Faculty of Electronics and Information Technology, Institute of Electronic Systems, \\ Warsaw University of Technology, 15/19 Nowowiejska, 00-665 Warsaw, Poland; \\ aleksander.augustyniak2.stud@pw.edu.pl \\ 2 Central Chamber for Telecommunication Measurement, National Institute of Telecommunications, \\ 1 Szachowa, 04-894 Warsaw, Poland; m.zdanowicz@il-pib.pl \\ * Correspondence: t.osuch@elka.pw.edu.pl
}

Citation: Augustyniak, A.; Zdanowicz, M.; Osuch, T. Self-Similarity Properties of Complex Quasi-Periodic Fibonacci and Cantor Photonic Crystals. Photonics 2021, 8, 558. https://doi.org/10.3390/ photonics 8120558

Received: 8 November 2021

Accepted: 4 December 2021

Published: 7 December 2021

Publisher's Note: MDPI stays neutral with regard to jurisdictional claims in published maps and institutional affiliations.

Copyright: (c) 2021 by the authors. Licensee MDPI, Basel, Switzerland. This article is an open access article distributed under the terms and conditions of the Creative Commons Attribution (CC BY) license (https:/ / creativecommons.org/licenses/by/ $4.0 /)$.

\begin{abstract}
In this paper, the influence of structural modifications on basic quasi-periodic $(\mathrm{QP})$ photonic crystals (PhC's) on self-similarity feature in their spectral responses is examined. Investigated crystals are chosen based on a present knowledge on the QP crystals, and are classified according to their structure. One of the QP crystals considered for the calculations is a concatenation, Fibonacci structure. It characterizes with a self-similar spectra for its different orders, which means, that the spectral shape repeats itself and can be partially identical for a different orders of the Fibonacci QP crystal. The calculations were also performed for the fractal structure, based on a Cantor QP crystal. Just as for the case of the Fibonacci structure, it characterizes with a self-similar spectra for different orders of the structure. Considered photonic devices are next put through simple modification operations by multiplication, conjugation or mirror reflection. Resulting, modified structures are used for the calculations of their spectral response. Results show, that the self-similarity of the spectra is not affected by performed modifications, and thus spectral response of QP PhC can be designed without losing this feature. Moreover the regular expansion of the repeated central part of the spectrum that appears in higher-order Fibonacci QP PhC spectra (due to the self-similarity) with the increase Fibonacci crystal order is presented here for the first time.
\end{abstract}

Keywords: one dimensional photonic crystals; self-similarity; quasi-periodic structures; transfer matrix method

\section{Introduction}

Photonic crystal devices have dominated the optical research in recent years. This substantial interest is justified by the incredible elasticity of such systems. Apart from the naturally occurring examples of photonic crystals, man-made structures were researched as far as half an age ago, when thin-film dielectric stacks were used as extremely effective optical filters [1]. Later, Yablonovitch proposed that the three-dimensional periodic structures could exhibit a range of frequencies, in which light could not propagate in any direction thru the structure [2]. This range of forbidden electromagnetic (EM) frequencies, or the photonic band-gap, allows for a strong confinement of EM fields, and moreover, for stopping an unwanted spontaneous emission in the forbidden frequency range. It was proven, that small disturbances in the structure do not destroy the properties of photonic crystals [3], which opened the way for multitude of applications. Nowadays, photonic crystals are utilised in laser systems [4-6], as efficient optical sensors [7-9], including complete lab-on-chip devices $[10,11]$. Photonic devices allow for an exceptional control of light, and therefore are utilised as negative refraction media [12], for the self-collimation [13] and light bending [14].

It is a well established fact, that the three-dimensional photonic structures demand excellent capabilities in matter manipulation, especially for the operation in the optical 
frequency range. For all the purposes, it is much more efficient to utilize two-dimensional structures, as the possible manufacturing methods are extremely precise and reliable. Moreover, those methods offer much wider variety of accessible materials [15]. All things considered, the fabrication is the most time consuming and cost ineffective process in the photonic crystals research. Due to the above, testing of a new ideas with a cheap and reliable computational tools is the first step towards realisation of fully functional devices. One of the most complex, and therefore hard to fabricate, examples of photonic devices are those, which contain quasi-periodic configuration of two or more optical materials. In this work, one-dimensional transfer matrix method (TMM) is applied to analyze examples of the photonic crystals with quasi-periodic Fibonacci, and fractal Cantor structures. In TMM method, the incident, reflected and transmitted waves are related to each other by means of matrices, which contain Fresnel coefficients determined analytically, and matrices representing the wave transmission through the homogeneous layers $[16,17]$. The calculated EM plane-wave propagates at normal incidence to the surface of the structure. The method was implemented in the MATLAB computer programming environment. The Fibonacci structures are established according to the Fibonacci sequence. Starting from the two regions of lower $(\mathrm{L})$ and higher $(\mathrm{H})$ refractive indices, each consecutive added element is a sum of the two previous elements. The rules governing of the assembling of such a structure are described in detail in previously published works [18-22]. In these works it was shown, that for the appropriate scaling of the frequency in graphical representation of the data, one can observe the emergent self-similarity of the resulting spectral response. This spectral response of the investigated structures is strongly dependent on the materials used, as well as the media surrounding the quasi-periodic (QP) photonic crystal (PhC) [19,23]. Cantor structure, on the other hand, is an example of the self-similar, fractal photonic crystal. The Cantor structure is established by division of the base element into three equal sections, and replacement of the middle section by another material. Starting from a uniform section of the higher $(\mathrm{H})$ refractive index, first order of the Cantor structure is established by division of the whole section into three equal parts, and replacement of the middle section with a material with the lower $(\mathrm{L})$ refractive index. Higher orders of the structure are created by a similar operation-each $\mathrm{H}$ part of the resulting structure is divided in three equal sections, with middle part replaced by $L$ material. All the resulting $L$ sections remain unaffected by the process. Good examples of the Cantor structures and their spectral response are presented in previously published works [24-26]. Both mentioned basic examples of QP crystals manifest self-similarity in observed spectral response. The clue difference in these spectra being: the order of the structure for which the similarity is observed, as well as the frequency range of the similar parts of the spectra $[18,23,24]$. There are some reports on possible additional modifications on the QP crystals $[20,27,28]$, which affect the spectral response of the resulting composite. In this paper the influence of the operations performed on the basic QP PhC on the resulting spectral responses, especially self-similarity feature, is examined. Considered modifications include: mirror reflection, conjugation and multiplication of the base structure. Two classes of QP PhC structures, i.e., concatenation (Fibonacci) and fractal (Cantor) are taken into consideration.

\section{Materials and Methods}

In the research presented here, Transfer Matrix Method $[16,17]$ was deployed for the purpose of the analysis of the spectral response of the quasi-periodic, one-dimensional photonic crystals. Structures under investigation were selected based on the method of their formation. In the most fundamental level, those can be classified as follows:

- concatenation structure-in which successive elements are created on the basis of the structure of the lower order. In case of the work presented here, the first two elements were defined as $S_{1}=\mathrm{L}, S_{2}=\mathrm{H}, S_{3}=S_{2}+S_{1}=\mathrm{HL}, S_{4}=S_{3}+S_{2}=\mathrm{HLH} .$. $S_{n}=S_{n-1}+S_{n-2}$ for $n \geq 3$. Resulting quasi-periodic structure fulfills the Fibonacci sequence, and is further referred to as a Fibonacci structure (Fib $b_{n}$, where $n$ is the 
order of the structure). The example of the Fibonacci structure of the eighth order is presented in Figure 1a.

- fractal structure-in which successive elements are formed in an iterative algorithm, by transforming each layer of the structure in a strictly defined manner. Cantor structure, described in detail in a previous section is an example of the fractal. In case of the work presented here, the 0th order of the structure is defined as a uniform slab of material $\mathrm{H}$ with higher refractive index. The algorithm for the creation of the higher orders of the Cantor structure ( $\mathrm{Can}_{n}$, where $n$ is the order of the Cantor structure) is very straight forward. To create Cantor structure of the first order $\left(\mathrm{Can}_{1}\right)$, the length of the $\mathrm{H}$ material is divided in three equal sections, and the middle section is then replaced with the $\mathrm{L}$ material with lower refractive index. For the creation of the $n$th order of the Cantor structure, the procedure is repeated recursively for each $\mathrm{H}$ section of the Cantor structure from Can 1 to Can $n_{n}$. Every L section of each resulting order of the Cantor structure always remains unchanged. The example of the first three orders of the Cantor structures created by the above set of rules is presented in Figure 1b.

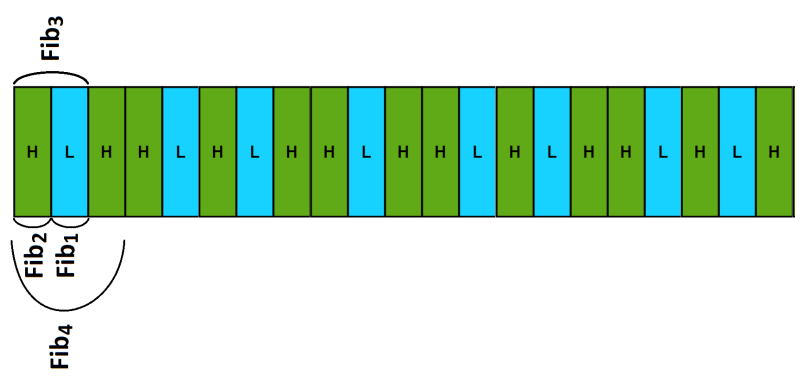

(a)
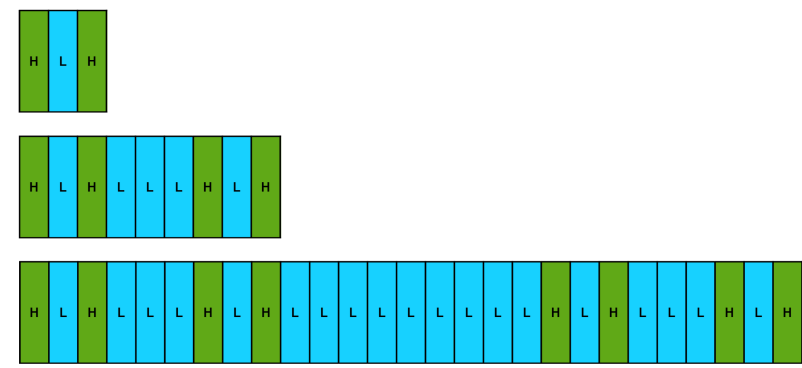

(b)

Figure 1. Schematic representation of the Fibonacci and Cantor structures used in the calculations. The complexity of the structures grows rapidly with the order of the structure, and for the sake of the clear images, only comparably low-order structures are presented here. (a) Example Fibonacci structure $\left(\mathrm{Fib}_{8}\right)$, with first four orders marked in an eighth order structure, (b) Cantor structure with the orders from one to three presented from the top to bottom.

In all the performed calculations, the value of the $\mathrm{H}$ material refractive index is taken to be 2.3, which corresponds to titanium dioxide $\left(\mathrm{TiO}_{2}\right)$. The refractive index for $\mathrm{L}$ material is taken to be $1.45\left(\mathrm{SiO}_{2}\right.$ silica glass) $[29,30]$. Both media are assumed to be lossless, optically passive, and dispersionless, for the sake of the calculations. The central frequency for the considered structures was chosen to be $v_{0}=600 \mathrm{THz}$, which corresponds to wavelength $\lambda_{0}=500 \mathrm{~nm}$. For the defined central wavelength $\lambda_{0}$, the thicknesses of the layers were chosen to be $d_{\mathrm{H}}=\frac{\lambda_{0}}{4 n_{\mathrm{H}}} \approx 54 \mathrm{~nm}$, and $d_{\mathrm{L}}=\frac{\lambda_{0}}{4 n_{\mathrm{L}}} \approx 86 \mathrm{~nm}$, so that effective path for the light in both the media is equal to $\lambda_{0} / 4$. In the case of the Cantor structures, the above thicknesses are used for the smallest features of the given order $n$, as presented in Figure $1 \mathrm{~b}$. In all the calculations, the structure is surrounded by air with refractive index $n_{0}=1$. The graphical data is presented in the frequency domain normalized to the central frequency (i.e., $\frac{v}{v_{0}}$ ), so the symmetries of the spectral response are well exposed. It was shown, that 
the centre of the symmetry for the QP crystal spectral response is at $v_{0}$, and at $2 v_{0}$ for the Fibonacci, and Cantor structures respectively. The self-similarity is observed for the spectra of the basic Fibonacci structures for the orders $n, n+6, n+12 \ldots$, where $n \geq 6$ is a natural number [23]. In case of the basic Cantor structures, self-similarity is observed in the spectral response of every order of the QP crystal [24].

Calculation method was verified by comparison against previously published works [23-26]. The validated code was then used to test new ideas of the QP PhC complex structures modified by mirror symmetry, conjugation and multiplication operations performed on different orders of Fibonacci and Cantor PhC's. Considered operations were implemented by adding modified structure of the certain order $n$ to the base structure according to a well defined algorithm.

The mirror symmetry is implemented by establishing the plane of symmetry for the QP structure at its end, and performing the modification operation according to the Figure 2a. In simple terms, modified structure is a sum of itself and its reflection at the mirror symmetry plane. Resulting structure is then codified with letter $R$ (e.g., second order Fibonacci structure with mirror operation is referred in the text as $R\left(F i b_{2}\right)$ ). The conjugated structure is created by exchange of every $\mathrm{H}$ layer by $\mathrm{L}$, and every $\mathrm{L}$ by $\mathrm{H}$ respectively in a base structure, and combining the base with its conjugation. The conjugated structure is codified by letter $C$, and the whole process is shown schematically in Figure 2b. The example of the second order Fibonacci structure with conjugation (base structure + negative structure) is referred in the text as $C\left(F i b_{2}\right)$ The last operation performed on the investigated structures is multiplication. It is achieved by simple addition of a repeated structure without alterations at the end of the base structure. The multiplication process is presented schematically in Figure 2c. The example of a second order Fibonacci structure modified by multiplication is referred in the text as $K\left(F i b_{2}\right)$, where $K$ is the number of repetitions of the base structure.

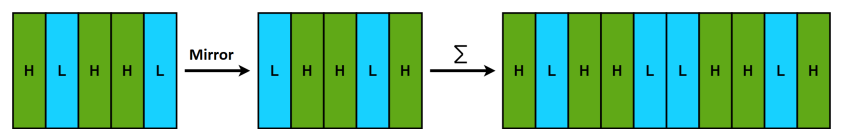

(a) Mirror symmetry.

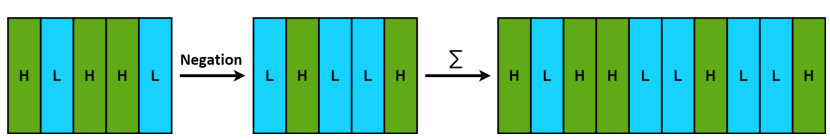

(b) Conjugated structure.

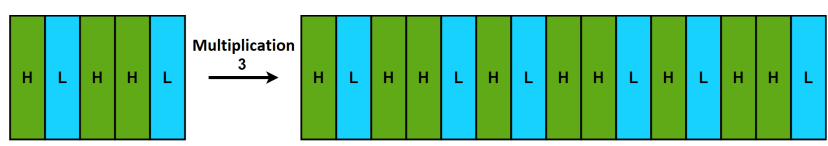

(c) Repeated structure.

Figure 2. General rule for the creation of the complex QP structures by modification operations: (a) mirror symmetry, (b) conjugation, (c) multiplication.

\section{Results}

The calculation results presented in Figure 3 point to an interesting quality of the resulting spectra. For each $n+6$ increment in the complex Fibonacci structure, the reported scaling factor of the frequency axis stays the same, as in previously reported basic Fibonacci QP PhC [23]. In case of the work presented here, it was calculated as the ratio of the spectral distance between two outer maximums contained within an orange frame visible in Figure 3: between $F i b_{8}$ and $F i b_{14}$; between $F i b_{14}$ and $F i b_{20}$; and so on. For every instance, it was estimated to be close to 26, which is consistent with the previous reports. However, it was also observed, that for the increased order of the Fibonacci structure, the similar part of the spectrum repeated in the higher order structure contains the original part of the spectra (orange frame in Figure 3, and the additional side-bands (green and violet 
frame in Figure 3). The situation repeats with every +6 increment of the order of the Fibonacci crystal. The estimate for the broadening factor of the similar part of the spectra was achieved by comparison of the spectral distance between the peaks furthest from the centre frequency ( 1 on the normalised frequency axis) contained in the colored frames in Figure 3. Taking all the above into account, the broadening factor for the similar part of the spectrum was calculated to be $\sim 6$. Combining the frequency axis scaling factor with the broadening factor of the similar part of the spectrum, gives the estimate for the spectral range of the similar part of the spectrum for the next +6 order of the Fibonacci structure. If the spectral width of the central part of the spectra of the Fib $b_{8}$ crystal is measured to be $\Delta \omega$ (orange frame Figure 3), then the similar part of the spectra repeated in $F_{i b} b_{14}$ structure will have the spectral width of $\Delta \omega / 26$, but the part of the $F i b_{14}$ spectra repeated in $F i b_{20}$ is estimated to be $6 \Delta \omega / 26$ (green frame), and the whole repeated part in Fib $b_{20}$ crystal will have a spectral width of $6 \Delta \omega / 26^{2}$. The estimate of the spectral width in $F i b_{20}$ to be repeated in $F i b_{26}$ is again six times broader: $6^{2} \Delta \omega / 26^{2}$, and this estimate can be repeated for every next generation of the Fibonacci structure in a recursive manner.
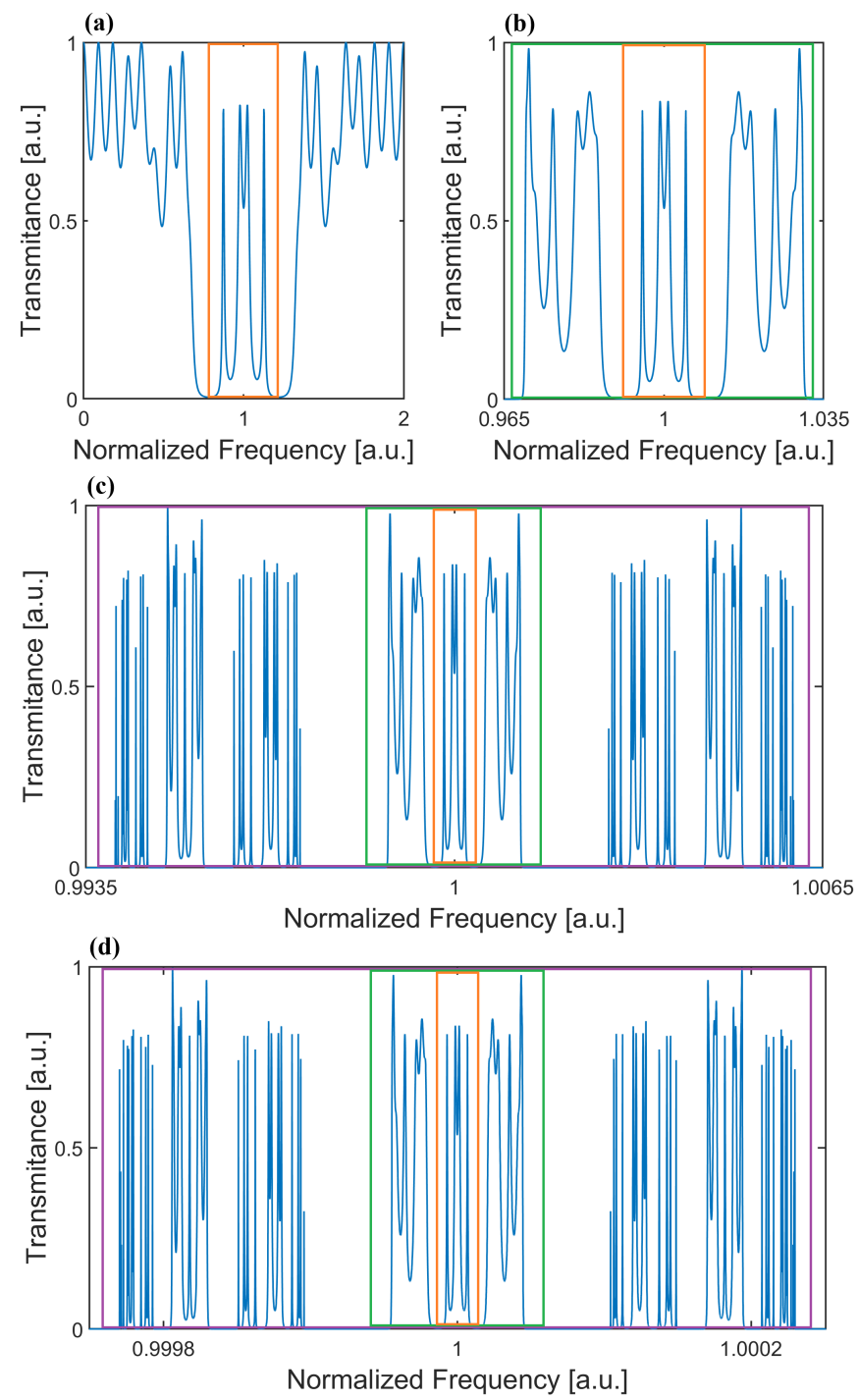

Figure 3. Scaling of the self-similar part of the spectrum with increasing order of the Fibonacci structure. (a) Fib 8 structure, (b) Fib 14 structure, (c) Fib 20 structure, (d) Fib 26 structure. The spectral width of the similar part of the calculated spectra for every order is compressed around $v_{0}$, which corresponds to 1 on the normalized axis. However, for each +6 order, the repeated part of the spectra broaden. 


\subsection{Modified Fibonacci Structures}

Calculated spectral responses for the complex Fibonacci structures with mirror symmetry is presented in Figure 4. It contains central (and similar) part of the spectra for the $R\left(F i b_{7}\right), R\left(F i b_{13}\right)$, and $R\left(F i b_{19}\right)$ quasi-periodic crystals. The arbitrary values of the transmittance (from 0 to 1 ) correspond to $0 \%$ and $100 \%$ transmission through the media, and the values presented here are the result of the calculations made with TMM model. In this model the transmittance is calculated as $T=\left|\frac{E_{\text {out }}}{E_{i n}}\right|^{2}$, and it is assumed that incident $E_{\text {in }}=1 \mathrm{~V} / \mathrm{m}$. In the figures, the normalized frequency is $\frac{v}{v_{0}}$, where $v_{0}=600 \mathrm{THz}$ corresponds to the wavelength $\lambda_{0}=500 \mathrm{~nm}$. In Figure 3 it is observed, that with every increment $n=6$ of the Fibonacci structure order, the similar part of the spectra preserves its shape, but contracts in the frequency domain. Apart from those, the shape of the calculated spectral response is well preserved in case of all the orders of the structures. The spectral response of the conjugated Fibonacci structure is presented in Figure 5. Similar to the case of the mirror symmetry, the self-similarity is observed for the structure orders separated by 6. It can be observed, that introducing different operations on a base structure results in a drastic change of the resulting spectral response. These effect might be utilised as a control tool in a design process of a certain optical filter. The shape in all the resulting spectra of the conjugated structures is well preserved. However, the width of the similar part of the spectrum is again contracting in the frequency domain with increasing order of the QP structure. The repetition of the Fibonacci structure gives interesting results with regards of the number of the repetitions $K$. The differences in the Fibonacci structure response of the same orders, but repeated $K=2$, and $K=3$ times are presented in Figure 6. All the calculations performed seem to point to a general rule, that for the even number of the repetitions $2 K$ ( $K$ is a natural number), the spectra always has a maximum for the centre frequency $v_{0}$. Moreover, for every odd number of the repetition $2 K+1$, the resulting spectra has a minimum at the central frequency $v_{0}$. Considering the differences in the spectral shapes of the multiplied Fibonacci structures, it can be observed, that the multiplication leads to a sequential splitting of the transmission peaks, similar to an analogical process reported for the case of the Cantor structures [24]. It can be seen, that for complex Fibonacci QP PhC with multiplication of the base structure, the self-similarity feature (with the order of +6 ) is also preserved.

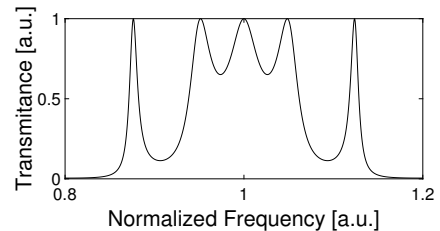

(a) $R\left(\mathrm{Fib}_{7}\right)$

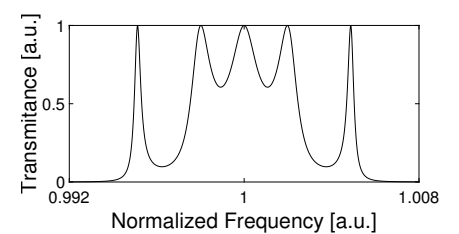

(b) $R\left(F i b_{13}\right)$

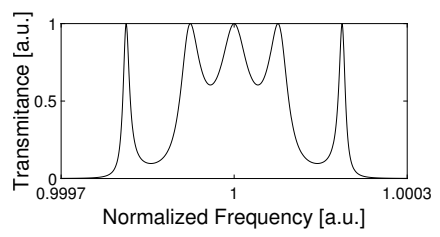

(c) $R\left(F i b_{19}\right)$

Figure 4. Self-similar spectra presented for the modified Fibonacci structure with mirror symmetry. Self-similarity is observed, just as in the case of standalone Fibonacci structure, between the orders differing by 6 .

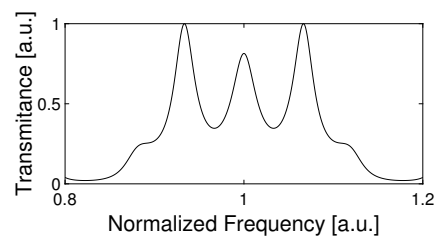

(a) $\mathrm{C}\left(\mathrm{Fib}_{7}\right)$

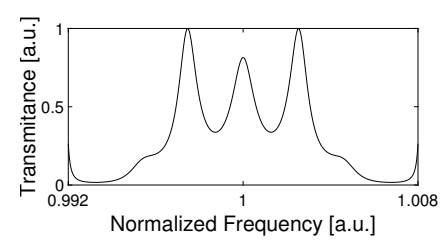

(b) $\mathrm{C}\left(\mathrm{Fib} \mathrm{b}_{13}\right)$

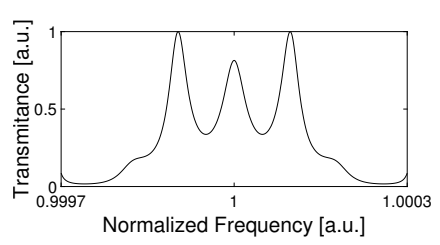

(c) $C\left(F i b_{19}\right)$

Figure 5. Self-similar spectra presented for the conjugated Fibonacci structure. Self-similarity observed for the orders separated by 6 . 


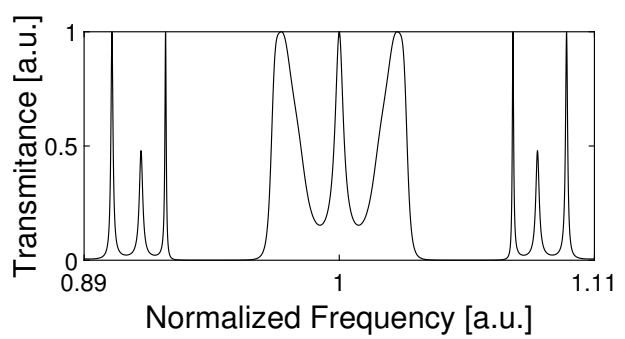

(a) $2\left(F i b_{10}\right)$

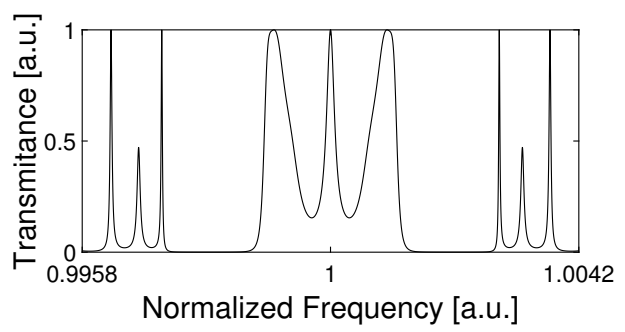

(c) $2\left(F i b_{16}\right)$

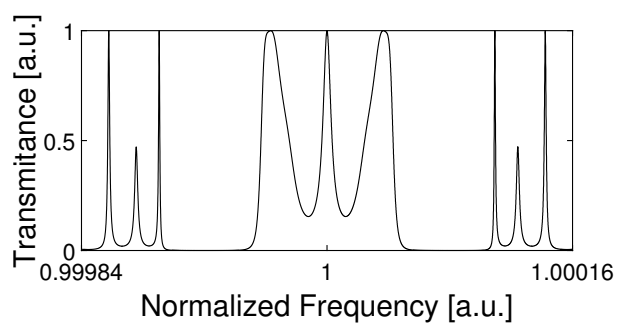

(e) $2\left(F i b_{22}\right)$

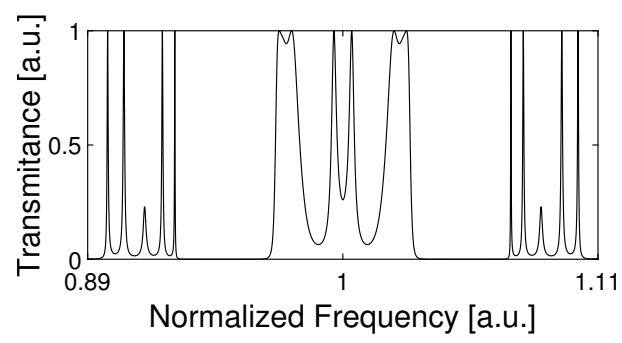

(b) $3\left(F i b_{10}\right)$

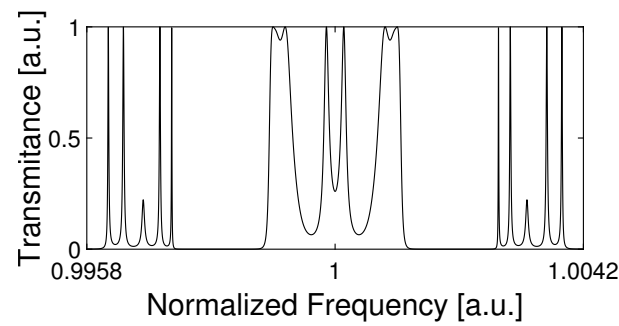

(d) $3\left(F i b_{16}\right)$

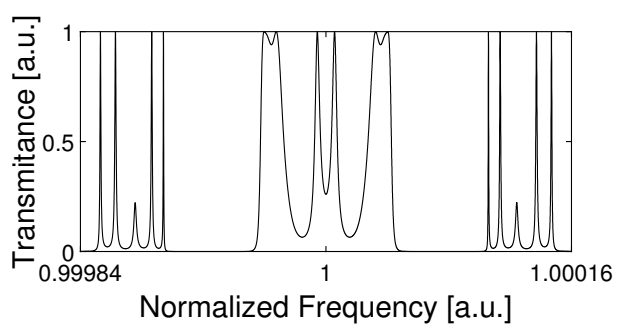

(f) $3\left(F i b_{22}\right)$

Figure 6. Self-similar spectra presented for the modified Fibonacci structure with multiplication of the base structure. Self-similarity observed for the orders separated by 6 .

\subsection{Modified Cantor Structures}

Due to the recursive algorithm for the construction of the Cantor structure, the resulting crystal always preserves the centre of symmetry in its middle. The consequence of the above is, that the modification by the repetition and the mirror image of the Cantor crystal yields the same structure. Creation of the modified Cantor structure by mirror symmetry or repetition are always equivalent. The calculation results for the repetition/mirror symmetry modification of the Cantor structures are presented in Figure 7. The self-similarity in the spectrum is observed between every order of the structure. The spectral width of the similar parts of the spectra contracts with increasing order of the structure as for the case of the Fibonacci structures. The conjugation of the Cantor structures yields similar results. The self-similar part of the spectrum is shown in Figure 8, and can observed for every order of the structure. The spectral width of the similar fragment of the spectrum also contracts with increasing order of the structure. However, the resulting shapes of the self-similar parts of all the Cantor structures spectra is not preserved as well as for the case of the Fibonacci structures presented in the previous subsection.

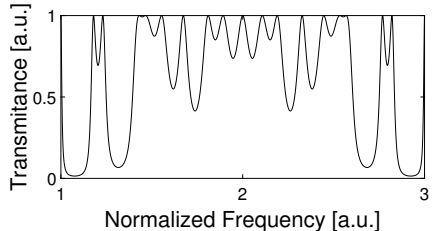

(a) $\mathrm{R}\left(\mathrm{Can}_{2}\right)$ or $2\left(\mathrm{Can}_{2}\right)$

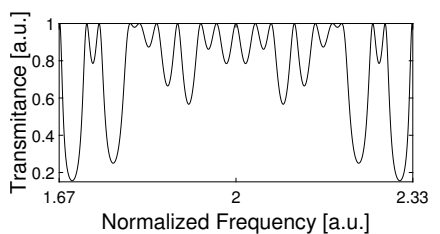

(b) $\mathrm{R}\left(\mathrm{Can}_{3}\right)$ or $2\left(\mathrm{Can}_{3}\right)$

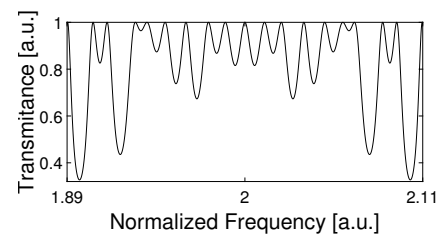

(c) $\mathrm{R}\left(\mathrm{Can}_{4}\right)$ or $2\left(\mathrm{Can}_{4}\right)$

Figure 7. Self-similar spectra calculated for the repetition/reflection of the Cantor structure. Selfsimilarity observed for the orders separated by 1 . 


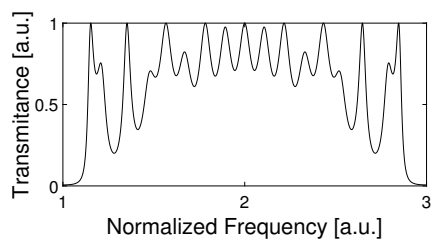

(a) $\mathrm{C}\left(\mathrm{Can}_{2}\right)$

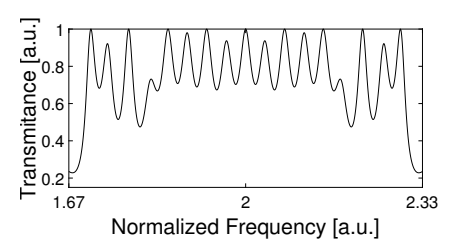

(b) $\mathrm{C}\left(\mathrm{Can}_{3}\right)$

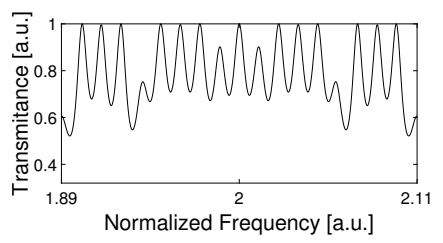

(c) $\mathrm{C}\left(\mathrm{Can}_{4}\right)$

Figure 8. Self-similar spectra presented for the conjugated Cantor structure. Self-similarity observed for the orders separated by 1 .

The calculations clearly show, that for the introduced modification operations on the basic QP structures, the self-similarity of the resulting spectra is always preserved. Moreover, the orders of the structures, at which the self-similar patterns are observed remain the same as in the case of the non-modified structures, for both: Fibonacci (6), and Cantor (1) structures alike. The centre of symmetry in both cases also remain unaffected by the modification of the structures, and stay at $v_{0}$, and $2 v_{0}$ for Fibonacci, and Cantor structure respectively.

\section{Discussion}

Calculations performed allow for a conclusive finding, that the proposed modifications (mirror symmetry, conjugation and multiplication) of the basic quasi-periodic structures do not affect the self-similarity of the resulting spectra. In fact, such operations do not affect the central frequency for the spectral response symmetry, as well as any other quantitative parameter of the resulting spectra (e.g., orders for the self-similar behaviour of the calculated spectra). The main advantage of using of an additional modification operations on QP crystals is the effect of such operations on the shape of the resulting spectra. The possible variations of such complex structures are virtually inexhaustible. Moreover, it was also shown for the first time, that together with the increasing order of the Fibonacci structure, the central part of the self-similar spectrum repeated in the higherorder Fibonacci QP PhC is broadened, and includes additional side bands, not present in the lower-order spectra. This phenomena together with the introduction of presented structural modifications of QP PhC's can be used for more conscious and predictable design of their spectral properties.

The work presented here is a first step towards systematic analysis of different variations of the modification operations on quasi-periodic structures, and their influence on the QP PhC spectral responses. By performing of a simple operations on a base structures in presented calculations, the present knowledge about the possible mechanisms governing the spectral response of QP structures is increased. From the point of view of the possible applications of such devices, which include optical filtering, especially multi-wavelength narrow-band optical filters, design and development of reflection calibration standards for experimental optics, or wavelength division multiplexing purposes; it is essential to provide meticulous analysis of the possible modifications, or combinations of QP structures, that can be used as a point of reference in the design process of the functional photonic devices. It is also worth to mention, that presented results provide the new knowledge on band structure modification of quasi-periodic low dimensional photonic devices. It shows another degree of freedom in predictable shaping of the QP PhC's spectral characteristics, where not only the increase of the order, but also the modifications of the basic structures is accounted for. In this way the QP PhC's can be widely optimized taking into account possibilities and limitations of current fabrication technologies. As already mentioned, the most efficient fabrication technology for such structures would be thin-film application by one of the well established methods, like magnetron sputtering, or plasma-enhanced chemical vapour deposition [15].It has been experimentally proven that above-mentioned technologies are suitable for thin-film layered structures fabrication using $\mathrm{TiO}_{2}$ and $\mathrm{SiO}_{2}$ materials [30]. The work presented here will be continued for the full classification of the possible operations and its effect on the resulting spectra. Results presented here point to a 
strong relation between the performed modification, kind of the base structure, number of repetitions in the modified structure, and the spectral response. Future plans include possible realisation of the simulated structures by a fruitful cooperation with a potential partner with an access to a suitable technology.

Author Contributions: Conceptualization, T.O.; methodology, A.A. and T.O.; software, A.A.; validation, A.A. and M.Z.; writing—original draft preparation, M.Z.; writing—review and editing, T.O. and A.A; visualization, A.A.; supervision, T.O. All authors have read and agreed to the published version of the manuscript.

Funding: This research received no external funding.

Institutional Review Board Statement: Not applicable.

Informed Consent Statement: Not applicable.

Data Availability Statement: Not applicable.

Conflicts of Interest: The authors declare no conflict of interest.

\section{Abbreviations}

The following abbreviations are used in this manuscript:

$\begin{array}{ll}\text { QP } & \text { quasi-periodic } \\ \text { PhC } & \text { photonic crystal } \\ \text { EM } & \text { electro-magnetic } \\ \text { TMM } & \text { transfer matrix method }\end{array}$

\section{References}

1. MacLeod, H.A. Thin-Film Optical Filters; Taylor \& Francis Ltd.: London, UK, 2021.

2. Yablonovitch, E. Inhibited Spontaneous Emission in Solid-State Physics and Electronics. Phys. Rev. Lett. 1987, 58, $2059-2062$. [CrossRef]

3. John, S. Strong localization of photons in certain disordered dielectric superlattices. Phys. Rev. Lett. 1987, 58, 2486-2489. [CrossRef] [PubMed]

4. Ginzburg, N.S.; Peskov, N.Y.; Sergeev, A.S.; Phelps, A.D.R.; Cross, A.W.; Konoplev, I.V. The use of a hybrid resonator consisting of one-dimensional and two-dimensional Bragg reflectors for generation of spatially coherent radiation in a coaxial free-electron laser. Phys. Plasmas 2002, 9, 2798-2802. [CrossRef]

5. Strauf, S.; Hennessy, K.; Rakher, M.T.; Choi, Y.S.; Badolato, A.; Andreani, L.C.; Hu, E.L.; Petroff, P.M.; Bouwmeester, D. Self-Tuned Quantum Dot Gain in Photonic Crystal Lasers. Phys. Rev. Lett. 2006, 96, 127404. [CrossRef] [PubMed]

6. VCSELs; Springer GmbH: Berlin/Heidelberg, Germany, 2012.

7. Nishijima, Y.; Ueno, K.; Juodkazis, S.; Mizeikis, V.; Misawa, H.; Tanimura, T.; Maeda, K. Inverse silica opal photonic crystals for optical sensing applications. Opt. Express 2007, 15, 12979. [CrossRef] [PubMed]

8. Descrovi, E.; Frascella, F.; Sciacca, B.; Geobaldo, F.; Dominici, L.; Michelotti, F. Coupling of surface waves in highly defined one-dimensional porous silicon photonic crystals for gas sensing applications. Appl. Phys. Lett. 2007, 91, 241109. [CrossRef]

9. Troia, B.; Paolicelli, A.; De, F.; Passaro, V.M.N. Photonic Crystals for Optical Sensing: A Review. In Advances in Photonic Crystals; IntechOpen Ltd.: London, UK, 2013. [CrossRef]

10. Shen, W.; Li, M.; Ye, C.; Jiang, L.; Song, Y. Direct-writing colloidal photonic crystal microfluidic chips by inkjet printing for label-free protein detection. Lab Chip 2012, 12, 3089. [CrossRef] [PubMed]

11. Zhang, Y.; Zhao, Y.; Zhou, T.; Wu, Q. Applications and developments of on-chip biochemical sensors based on optofluidic photonic crystal cavities. Lab Chip 2018, 18, 57-74. [CrossRef] [PubMed]

12. Cubukcu, E.; Aydin, K.; Ozbay, E.; Foteinopoulou, S.; Soukoulis, C.M. Negative refraction by photonic crystals. Nature 2003, 423, 604-605. [CrossRef] [PubMed]

13. Noori, M.; Soroosh, M.; Baghban, H. Self-Collimation in Photonic Crystals: Applications and Opportunities. Annalen Physik 2017, 530, 1700049. [CrossRef]

14. Zhang, Y.; Li, B. Photonic crystal-based bending waveguides for optical interconnections. Opt. Express 2006, 14, 5723. [CrossRef] [PubMed]

15. Martin, P. Handbook of Deposition Technologies for Films and Coatings: Science, Applications and Technology; Elsevier: Amsterdam, The Netherlands, 2010.

16. Yeh, P.; Yariv, A.; Hong, C.S. Electromagnetic propagation in periodic stratified media. I. General theory. J. Opt. Soc. Am. 1977, 67, 423. [CrossRef] 
17. Yariv, A.; Yeh, P. Electromagnetic propagation in periodic stratified media. II. Birefringence, phase matching, and X-ray lasers. J. Opt. Soc. Am. 1977, 67, 438. [CrossRef]

18. Kohmoto, M.; Sutherland, B.; Iguchi, K. Localization of optics: Quasiperiodic media. Phys. Rev. Lett. 1987, 58, 2436-2438. [CrossRef] [PubMed]

19. Gellermann, W.; Kohmoto, M.; Sutherland, B.; Taylor, P.C. Localization of light waves in Fibonacci dielectric multilayers. Phys. Rev. Lett. 1994, 72, 633-636. [CrossRef] [PubMed]

20. Mauriz, P.; Vasconcelos, M.; Albuquerque, E. Optical transmission spectra in symmetrical Fibonacci photonic multilayers. Phys. Lett. A 2009, 373, 496-500. [CrossRef]

21. $\mathrm{Wu}, \mathrm{J} . ; \mathrm{Gao}, \mathrm{J}$. Transmission properties of Fibonacci quasi-periodic one-dimensional superconducting photonic crystals. Optik 2012, 123, 986-988. [CrossRef]

22. Trabelsi, Y.; Ali, N.B.; Belhadj, W.; Kanzari, M. Photonic Band Gap Properties of One-dimensional Generalized Fibonacci Photonic Quasicrystal Containing Superconductor Material. J. Supercond. Nov. Magn. 2019, 32, 3541-3547. [CrossRef]

23. Vasconcelos, M.S.; Albuquerque, E.L.; Mariz, A.M. Optical localization in quasi-periodic multilayers. J. Phys. Condens. Matter 1998, 10, 5839-5849. [CrossRef]

24. Lavrinenko, A.V.; Zhukovsky, S.V.; Sandomirski, K.S.; Gaponenko, S.V. Propagation of classical waves in nonperiodic media: Scaling properties of an optical Cantor filter. Phys. Rev. E 2002, 65, 036621. [CrossRef] [PubMed]

25. Zhukovsky, S.V.; Lavrinenko, A.V.; Gaponenko, S.V. Spectral scalability as a result of geometrical self-similarity in fractal multilayers. Europhys. Lett. (EPL) 2004, 66, 455-461. [CrossRef]

26. Zhukovsky, S.; Lavrinenko, A. Spectral self-similarity in fractal one-dimensional photonic structures. Photonics Nanostruct. Fundam. Appl. 2005, 3, 129-133. [CrossRef]

27. Huang, X.; Wang, Y.; Gong, C. Numerical investigation of light-wave localization in optical Fibonacci superlattices with symmetric internal structure. J. Phys. Condens. Matter 1999, 11, 7645-7651. [CrossRef]

28. Zhukovsky, S.V. Perfect transmission and highly asymmetric light localization in photonic multilayers. Phys. Rev. A 2010, 81, 053808 . [CrossRef]

29. Bouazzi, Y.; Kanzari, M. Optical Fabry-Perot filter based on photonic band gap quasi-periodic one-dimensional multilayer according to the definite Rudin-Shapiro distribution. Opt. Commun. 2012, 285, 2774-2779. [CrossRef]

30. Jena, S.; Tokas, R.; Sarkar, P.; Misal, J.; Haque, S.M.; Rao, K.; Thakur, S.; Sahoo, N. Omnidirectional photonic band gap in magnetron sputtered $\mathrm{TiO}_{2} / \mathrm{SiO}_{2}$ one dimensional photonic crystal. Thin Solid Films 2016, 599, 138-144. [CrossRef] 